\title{
Comparative Studies on the Pre-enrichment Media for the Isolation of Salmonella in Fish Meal Samples
}

\author{
Kazuaki Saheki*
}

(Accepted January 19, 1987)

\begin{abstract}
Comparative studies were carried out on the pre-enrichment media for the isolation of salmonellae from fish meal samples.

In Experiment I, the following three media: buffered saline solution (BS), buffered peptone water (BPT) and Enterobacteriaceae enrichment mannitol broth (EEM) were compared with their selectivities for qualitative pre-enrichment media.

In Experiment II, seven pre-enrichment media, including the directly employed selenite broth for the quantitative estimation of salmonellae were evaluated for their effectiveness, using $S$. typhimurium suspension and the fish meal sample inoculated with the same organism.

Results obtained can be summarized as follows: In Exp. I, 39 commercial fish meal samples were examined to compare selectivities of 3 pre-enrichment media, and BS exhibited the highest positive rates being $24.9 \%$, followed by BPT $(23.0 \%)$, while the positive rates of EEM were as low as $11.1 \%$. In Exp. II, it was found that $0.1 \%$ meat extract containing BS (BSM), $0.1 \%$ peptone water $(0.1 \% \mathrm{PW})$ and nutrient broth gave high recoveries, followed by a half concentration of BPT. However, BPT, EEM and the directly employed selenite broth gave rather poor recoveries as compared with the former four media. As a selective culture medium of salmonellae, Hajina's tetrathionate broth was found to give higher recoveries than the selenite broth.

Based on the aforementioned results, the author wishes to propose the following procedures for the isolation and estimation of salmonellae from fish meal samples; BS will be employed as a pre-enrichment culture medium at $37^{\circ} \mathrm{C}$, and for the qualitative test, Hajina's tetrathionate broth will be used as a selective enrichment medium at $43^{\circ} \mathrm{C}$, in addition, for the quantitative estimation, BSM is recommended as the pre-enrichment medium.
\end{abstract}

The annual production of fish meal in Japan amounts to approximately 900,000 tons, in addition, about 100,000 tons of fish meal are imported every year, especially from South East Asian countries. Almost all these fish meal are consumed for the preparation of feedstuffs including those for fish culturing. Salmonelosis of domestic animals and fowls is one of the serious public health problems all over the world. ${ }^{1,2)}$ Recently, in Japan, contaminations of cultured fish with salmonellae have been widely spread in various fish species, and much attention has been paid to the possibility of outbreaks of human Salmonella intoxication ${ }^{2>}$ by ingesting fresh fish meat and various fishery products. One of the important cause of animal salmonellosis or contamination of cultured fish with Salmonella is known to be directly associated with the consumption of polluted feeds.

It has been pointed out by many workers that an appropriate pre-enrichment culture prior to the inoculation to a selective culture medium would be fairly important in the isolation process of genus Salmonella from various samples. In the present report, comparative studies on the preenrichment media for the isolation of Salmonella from fish meal have been carried out.

\section{Experimental}

I. Comparison of pre-enrichment media for qualitative test.

\section{Materials and Methods}

\section{Fish Meal Samples:}

Samples were obtained principally from the three fish meal factories in Choshi, Chiba Prefecture, since this area is one of the three major fish meal producing areas, Kushiro (Hokkaido), Hachinohe (Aomori Pref.) and Choshi, in addition, this area is located at the nearest and convenient place from

* Tokyo University of Fisheries, Konan, Minato, Tokyo 108, Japan (佳伯和昭: 東京水産大学). 
Table 1. Composition of the pre-enrichment media tested in Experiment I

1. Buffered Saline Solution (BS)

$\mathrm{NaCl}, \ldots \ldots \ldots \ldots \ldots \ldots \ldots \ldots \ldots, \ldots, \ldots \ldots \ldots$

Distilled Water (D. W.). . . . . . . . 1,000 ml

Buffer solution................. $1.25 \mathrm{ml}$

Buffer solution: Dissolve $34.0 \mathrm{~g}$ of $\mathrm{KH}_{2} \mathrm{PO}_{4}$ in $500 \mathrm{~m} l$ of $\mathrm{D}$. W., adjusted to $\mathrm{pH} 7.2$ with $1 \mathrm{~N} \mathrm{NaOH}$ and make up to one litter with $\mathrm{D}$. W.

2. Buffered Peptone water (BPT)

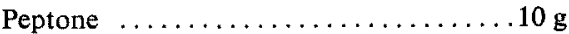

$\mathrm{NaCl} \ldots \ldots \ldots \ldots \ldots \ldots \ldots \ldots . \ldots . \ldots . \ldots . \ldots$

$\mathrm{Na}_{2} \mathrm{HPO}_{4} \cdot 12 \mathrm{H}_{2} \mathrm{O} \ldots \ldots \ldots \ldots \ldots \ldots . . .6 \mathrm{~g}$

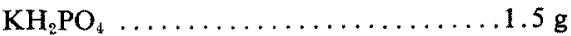

D. W. ...................

Tergitol 7 (Sigma) ..............6 $\mathrm{m} l$

3. Enterobacteriaceae Enrichment Mannitol broth

"Eiken" (EEM)

Triptone $. \ldots \ldots \ldots \ldots \ldots \ldots \ldots \ldots \ldots . \ldots \ldots$

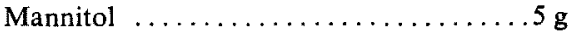

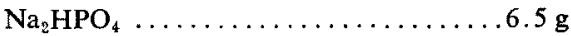

Ox gall powder .............20 g

Brilliant Green............... $0.0135 \mathrm{~g}$

D. W. ................., $1,000 \mathrm{ml}$

(pH 7.2)

Each medium is autoclaved at $121^{\circ} \mathrm{C}$ for 15 minutes.

our laboratory in Tokyo.

A total of 39 samples were collected in the period from October 1981 to December 1982.

\section{Pre-enrichment culture:}

Samples were inoculated in the following three media, buffered saline solution (BS), ${ }^{4)}$ buffered peptone water with $0.6 \%$ Tergitol $(\mathrm{BPT})^{5, e)}$ and Enterobacteriaceae enrichment mannitol broth (EEM), "Eiken," "7) and their compositions were shown in Table 1.

\section{Enrichment media:}

Selenite broth "Eiken" and Hajna's tetrathionate broth "Eiken" for their selectivities of Salmonella organisms were compared with each other.

\section{Selective plating medium:}

Desoxycholate-hydrogen sulfide-lactose agar (DHL) "Eiken" plate was used as a selective plating medium.

\section{Other media employed ${ }^{7}$ :}

The SIM medium was used for the tests of indole production, IPA (indole-pyruvic acid) and $\mathrm{H}_{2} \mathrm{~S}$ formation and motility.

For the tests of $\mathrm{H}_{2} \mathrm{~S}$ production, and glucose, lactose and sucrose fermentation, triple sugars

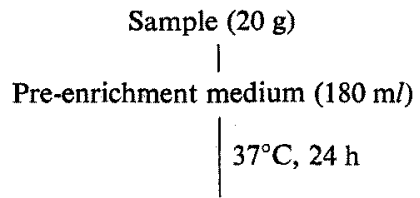

Selective medium

$$
43^{\circ} \mathrm{C}, 24 \mathrm{~h}
$$

DHL plate

$$
37^{\circ} \mathrm{C}, 24 \mathrm{~h}
$$

Several typical colonies

Biochemical tests

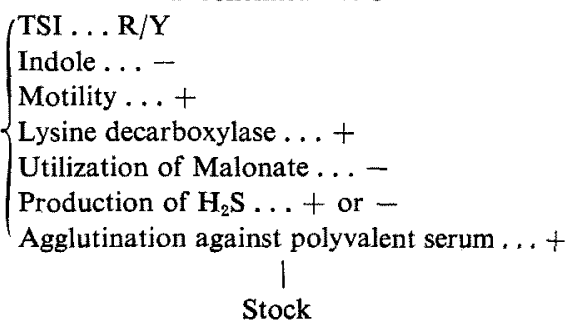

Fig. 1. Isolation scheme for salmonellae from fish meal samples.

iron agar (TSI) medium was used.

For the lysine decarboxylase test, lysine decarboxylation test medium, and for the malonate utilization test, malonate medium were employed, respectively.

All these media were purchased from Eiken Co., Tokyo, Japan.

\section{Procedure:}

Each sample was consisted of $1,500 \sim 2,000 \mathrm{~g}$ of fish meal in a polyethylene bag, from which about $200 \mathrm{~g}$ was taken to conduct microbiological test, and the remainder offered to another experiments.

The isolation scheme for genus Salmonella is illustrated in Figure 1. Twenty gram aliquots of a fish meal sample were inoculated into each $180 \mathrm{~m} l$ portion of two or three different pre-enrichment media, which were subjected to incubation at $37^{\circ} \mathrm{C}$ for $18-24 \mathrm{~h}$. After vigorously shaken, each $1 \mathrm{~m} l$ aliquot of these culture fluids was transplanted into 5 tubes containing $10 \mathrm{~m} l$ of selenite broth, and in the same numbers of Hajna's tetrathionate broth tubes, and these tubes were incubated in a water bath at $43^{\circ} \mathrm{C}$ for $18-24 \mathrm{~h}$.

From the test tubes showing positive growth, each one loop-full of the culture was streaked on a DHL plate, which was then incubated at $37^{\circ} \mathrm{C}$ 
Table 2. Comparison of pre-enrichment media and selective media for the isolation of salmonellae from fish meal samples

\begin{tabular}{|c|c|c|c|c|c|c|c|c|}
\hline \multirow[b]{3}{*}{ No. } & \multirow[b]{3}{*}{ Sample } & \multicolumn{2}{|c|}{ BS } & \multicolumn{2}{|c|}{$\begin{array}{l}\text { Pre. med. } \\
\text { BPT }\end{array}$} & \multicolumn{2}{|c|}{ EEM } & \multirow{3}{*}{ Note } \\
\hline & & \multicolumn{6}{|c|}{ Select. med. } & \\
\hline & & Sel.*1 & Tet. $* 2$ & Sel. & Tet. & Sel. & Tet. & \\
\hline 1 & D8204A & $5 / 5^{* 3}$ & $5 / 5$ & $4 / 5$ & $5 / 5$ & $0 / 5$ & $0 / 5$ & \\
\hline 2 & D8207A & $5 / 5$ & $5 / 5$ & $0 / 5$ & $0 / 5$ & $0 / 5$ & $0 / 5$ & \\
\hline 3 & $\mathrm{D} 8207 \mathrm{C}$ & $4 / 5$ & $5 / 5$ & $4 / 5$ & $5 / 5$ & $5 / 5$ & $5 / 5$ & \\
\hline 4 & A8207D & $5 / 5$ & $5 / 5$ & $0 / 5$ & $0 / 5$ & $0 / 5$ & $0 / 5$ & \\
\hline 5 & A8208 & $0 / 5$ & $4 / 5$ & $4 / 5$ & $5 / 5$ & $0 / 5$ & $3 / 5$ & \\
\hline 6 & D8209A & $5 / 5$ & $4 / 5$ & $4 / 5$ & $4 / 5$ & $5 / 5$ & $5 / 5$ & \\
\hline 7 & D8209B & $5 / 5$ & $5 / 5$ & $5 / 5$ & $5 / 5$ & $5 / 5$ & $5 / 5$ & \\
\hline 8 & $\mathrm{D} 8210 \mathrm{C}$ & $0 / 5$ & $0 / 5$ & $5 / 5$ & $4 / 5$ & $0 / 5$ & $0 / 5$ & \\
\hline 9 & D $8210 \mathrm{C}$ & $3 / 5$ & $1 / 5$ & $0 / 5$ & $0 / 5$ & - & - & Reexamined sample \\
\hline 10 & $\mathrm{~B} 8210 \mathrm{D}$ & $3 / 10$ & $9 / 10$ & $0 / 10$ & $9 / 10$ & - & - & \\
\hline 11 & D8211A & $0 / 10$ & $0 / 10$ & $0 / 5$ & $5 / 5$ & - & - & \\
\hline 12 & $\mathrm{D} 8212 \mathrm{C}$ & $5 / 5$ & $4 / 5$ & $5 / 5$ & $0 / 5$ & - & - & \\
\hline 13 & A $8212 B$ & $5 / 5$ & $5 / 5$ & $5 / 5$ & $4 / 5$ & 一 & - & \\
\hline \multirow{2}{*}{\multicolumn{2}{|c|}{ Total of $13^{* 4}$ samples }} & $45 / 75$ & $52 / 75$ & $36 / 70$ & $46 / 70$ & $15 / 40$ & $18 / 40$ & All Sel. $\quad 96 / 185(51.9 \%)$ \\
\hline & & $(60.0 \%)$ & $(69.3 \%)($ & $51.4 \%)($ & $65.7 \%)$ & $37.5 \%)($ & $45.0 \%)$ & All Tet. $116 / 185(62.7 \%)$ \\
\hline \multirow{3}{*}{\multicolumn{2}{|c|}{ Total of $39^{* 5}$ samples }} & $45 / 194$ & $52 / 196$ & $36 / 177$ & $46 / 179$ & $15 / 148$ & $18 / 150$ & All Sel. $\quad 96 / 519(18.5 \%)$ \\
\hline & & \multicolumn{6}{|c|}{$(23.2 \%)(26.5 \%)(20.3 \%)(25.7 \%)(10.1 \%)(12.5 \%)$} & All Tet. $116 / 525(22.1 \%)$ \\
\hline & & \multicolumn{2}{|c|}{$97 / 390(24.9 \%)$} & \multicolumn{2}{|c|}{$82 / 356(23.0 \%)$} & \multicolumn{2}{|c|}{$33 / 298(11.1 \%)$} & \\
\hline
\end{tabular}

*1 Selenite broth.

*2 Hajna's Tetrathionate broth.

*a Number of salmonellae positive tubes/Number of inoculated tubes.

* Total number of salmonellae positive samples.

*5 Total number of samples tested.

for 20-24 h. One to three colonies suspected of salmonellae per plate were picked up to submit biological and serological tests. An isolate was identified with genus Salmonella when it was coincided with the following typical biological characteristics, viz., indole production being -; IPA formation, -; motility, + ; fermentation of carbohydrates: glucose, +, lactose, -, and sucrose, - ; production of $\mathrm{H}_{2} \mathrm{~S}$, + or - ; lysine decarboxylase, +; malonate utilization, - ; and positive agglutination against polyvalent Salmonella antiserum. The isolates were further subjected to serological typing of Salmonella.

\section{Results}

Thirteen out of 39 fish meal samples including one reexamined sample and three environmental samples were found to be contaminated with salmonellae. The positive detection rates of Salmonella in the three different pre-enrichment cultures were summarized in Table 2, exhibiting that the BS gave the highest positive rates being $24.9 \%$ (97/390 tubes), followed by BPT, $23.0 \%$
$(82 / 356)$, and the figure of EEM was as low as $11.1 \%(33 / 298)$. No significant difference in the positive detection rates could be seen between those of BS and BPT, however, as shown in Table 1 , the component of $\mathrm{BS}$ is very simple compared with those of BPT.

Both selenite broth and Hajna's tetrathionate broth are commonly employed in Japan as the selective enrichment culture media for the examination of materials associated with Salmonella food poisoning. As shown in Table 2, it was found that the latter broth gave much higher positive rates than the former ones.

II. Comparison of pre-enrichment media for quantitative test.

In Experiment 1, it was revealed that a simple buffered saline solution (BS) gave excellent results as a pre-enrichment medium for the isolation of Salmonella from fish meal samples. In general, Salmonella positive samples are submitted to the quantitative test. In the case of qualitative test, a $20 \mathrm{~g}$ of fish meal sample is inoculated into a $180 \mathrm{~m} l$ of pre-enrichment medium, and this considerably large size of inoculum may 
Table 3. Composition of the pre-enrichment media tested in Experiment II

1. $0.1 \%$ Meat extract added Buffered Saline Solution (BSM)

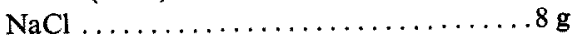

Meat ext. ..................

D. W. ................... 1,000 ml

Buffered Solution* ${ }^{*} \ldots \ldots \ldots \ldots \ldots 1.25 \mathrm{ml}$

2. 1/2 Buffered Peptone Water (1/2 BPW)

Peptone $\ldots \ldots \ldots \ldots \ldots \ldots \ldots \ldots \ldots . \ldots \ldots$

$\mathrm{NaCl} \ldots \ldots \ldots \ldots \ldots \ldots \ldots \ldots \ldots 2.5 \mathrm{~g}$

$\mathrm{Na}_{2} \mathrm{HPO}_{4} \cdot 12 \mathrm{H}_{2} \mathrm{O} \ldots \ldots \ldots \ldots \ldots \ldots \ldots 4.5 \mathrm{~g}$



D. W. .................. $1,000 \mathrm{ml}$

3. Tergitol added Buffered Peptone Water (BPT)

Peptone ..................... $10 \mathrm{~g}$

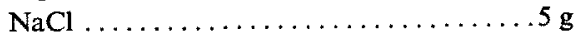

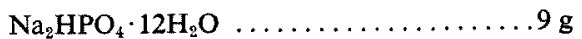

$\mathrm{KH}_{2} \mathrm{PO}_{4} \ldots \ldots \ldots \ldots \ldots \ldots \ldots \ldots \ldots \ldots \ldots \ldots \ldots \ldots$

D. W. ...................,

Tergitol 7 (Sigma) ............. $6 \mathrm{ml}$

4. Enterobacteriaceae Enrichment Mannitol Broth "Eiken" (EEM)

Triptone .................... $10 \mathrm{~g}$

Mannitol .....................

$\mathrm{Na}_{2} \mathrm{HPO}_{4} \ldots \ldots \ldots \ldots \ldots \ldots \ldots \ldots \ldots .5 \mathrm{~g}$

Ox gall powder ...............20 g

Brilliant Green ................ $0.0135 \mathrm{~g}$

D. W. ................ $1,000 \mathrm{ml}$

pH 7.2

5. Selenite Broth "Eiken" (Sel)

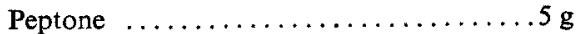

Lactose ................... $4 \mathrm{~g}$

Na-Selenite $\ldots \ldots \ldots \ldots \ldots \ldots \ldots \ldots \ldots, 4 \mathbf{g}$

$\mathrm{K}_{2} \mathrm{HPO}_{4} \ldots \ldots \ldots \ldots \ldots \ldots \ldots \ldots \ldots \ldots \ldots$

D. W. ................. $1,000 \mathrm{ml}$

$\mathrm{pH} 7.2$

6. Nutrient Broth "Eiken" (NB)

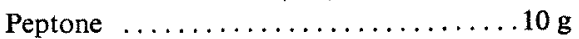

Meat extract $\ldots \ldots \ldots \ldots \ldots \ldots \ldots \ldots \ldots .6 \ldots$

$\mathrm{NaCl} \ldots \ldots \ldots \ldots \ldots \ldots \ldots \ldots \ldots . \ldots \ldots$

D. W. .................. $1,000 \mathrm{ml}$

pH 7.0

7. $0.1 \%$ Peptone Water $(0.1 \%$ PW)

Peptone $\ldots \ldots \ldots \ldots \ldots \ldots \ldots \ldots \ldots \ldots \ldots$

D. W. ................... $1,000 \mathrm{~m} l$

$\mathrm{pH}$ not adjusted

* See Table 1.

enhance the growth of salmonellae in the medium due to the presence of fairly large amounts of soluble matters in the test sample. In contrast, the inoculum size for quantitative test is apparently small compared with the qualitative test, e.g., a series of samples weighing $0.1,0.01,0.001 \mathrm{~g}$ or less are inoculated into $9 \mathrm{ml}$ portions of a pre-enrichment medium, respectively.

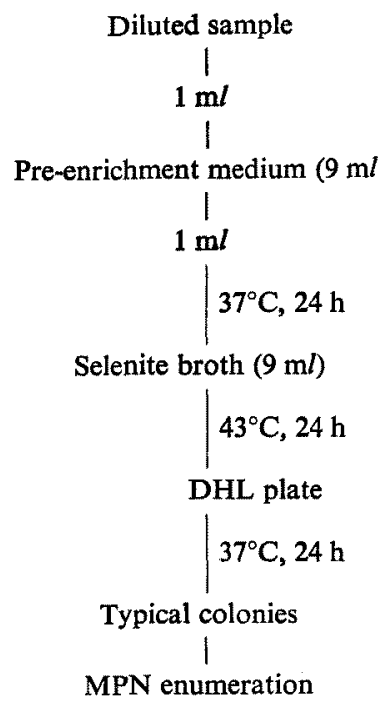

Fig. 2. Scheme for the quantitative pre-enrichment media employed in Exp. II.

BS was used as a pre-enrichment medium for the qualitative test, however, this medium contains slight amount of nutrients requing for the growth of salmonellae. Therefore, in the experiment herein, 7 different pre-enrichment media for the enumeration of salmonellae in fish meal samples were compared with one another.

\section{Materials and Methods}

\section{Test organism:}

Salmonella typhimurium (TUF-S1) was employed as the test organism.

Sample:

a) Cell suspensions A 24 h culture of the test organism grown in nutrient broth at $37^{\circ} \mathrm{C}$ was diluted with BS to make about $10^{3}$ magnitude of cells $/ \mathrm{m} l$, and distributed it in five tubes each containing $20 \mathrm{~m} l$ of this suspension.

b) Fish meal A cell suspension containing approximately $10^{\circ} \mathrm{cells} / \mathrm{m} l$ was prepared in the same manner described above, and a $0.2 \mathrm{~m} /$ aliquot of the suspension was added to a $20 \mathrm{~g}$ of Salmonella free fish meal sample with vigorously shaking to make uniform distribution.

The artificially contaminated fish meal sample thus prepared was divided into five portions which were distributed in sterile $200 \mathrm{ml}$ Erlenmeyer flasks, respectively.

These cell suspensions and fish meal samples to be tested were stored in a freezer at $-20^{\circ} \mathrm{C}$ before analyses. 
Table 4. Changes in the counts of $S$. typhimurium during frozen storage in buffered saline solution, and comparison of pre-enrichment media for the recoveries of test organism

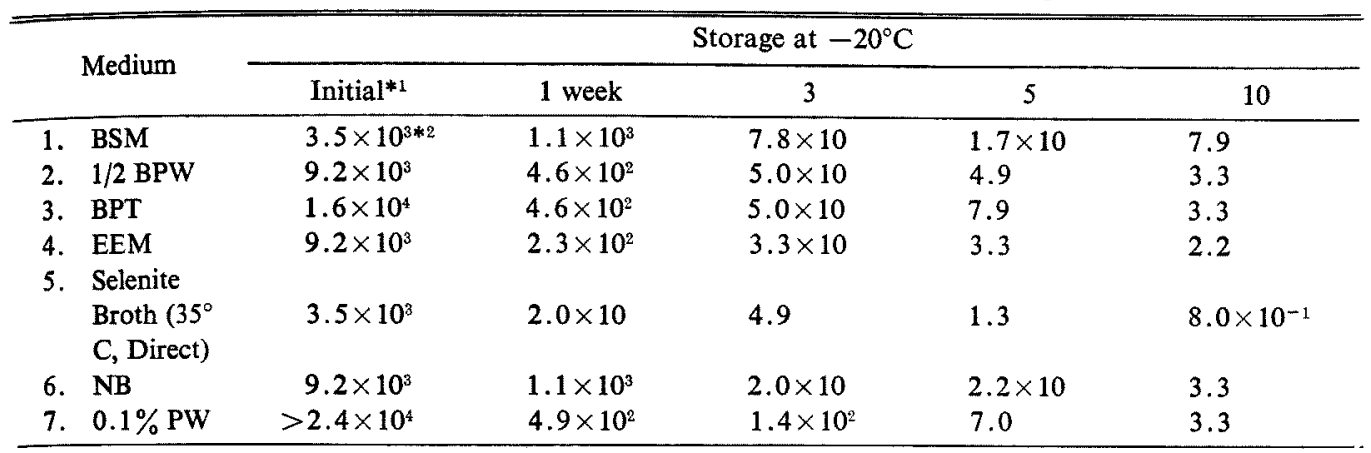

-1 Initial bacterial number of the solution: $9.2 \times 10^{3} / \mathrm{m} /$ cells (MPN in Nutrient Broth)

*2 Number of bacterial cells (MPN/mi).

Pre-enrichment media examined:

1. $0.1 \%$ Meat extract containing buffered saline solution (BSM)

2. A half concentration of buffered peptone water $(1 / 2 \mathrm{BPW})$

3. Buffered peptone water added with Tergitol (BPT)

4. Enterobacteriaceae enrichment mannitol broth, "Eiken" (EEM)

5. Selenite broth, "Eiken" (direct culture method) (Sel)

6. Nutrient broth, "Eiken" (NB)

7. $0.1 \%$ Peptone water $(0.1 \% \mathrm{PW})$

Compositions of these pre-enrichment media are shown in Table 3.

\section{Procedure:}

The enumeration of Salmonella in the two types of test samples were conducted according to the procedure as illustrated in Fig. 2, and results were expressed in terms of most probable number (MPN) (5 tubes method), viz., these two types of samples were prepared five replications respectively for the analyses, and they were stored at $-20^{\circ} \mathrm{C}$. Prior to the analysis, test samples were thawed at room temperature, then $1 \mathrm{~m} l$ was taken from the cell suspension sample, and $1 \mathrm{~g}$ was taken from the fish meal sample, and these were diluted with BS by the ten fold serial dilution, and then appropriately diluted samples were inoculated into seven different pre-enrichment media to be tested, and these inoculated media were incubated at $37^{\circ} \mathrm{C}$ for $24 \mathrm{~h}$. A $1 \mathrm{ml}$ aliquot of culture from the growth positive tube was then transplanted to selenite broth tube, and cultured at $43^{\circ} \mathrm{C}$ for $24 \mathrm{~h}$ in a water bath. The selenite broth tubes showing positive growth were streaked on DHL agar plates, which were incubated at $37^{\circ} \mathrm{C}$ for $24 \mathrm{~h}$. When typical Salmonella colonies appeared on DHL agar plates, the corresponding selenite broth tubes were regarded as Salmonella positive, and based on the number of positive tubes, MPN values were calculated, respectively. These MPN values obtained with different pre-enrichment media were compared with one another to evaluate the usefulness regarding the quantitative estimation of Salmonella in fish meal samples.

Changes of Salmonella cell numbers in test samples were determined immediately after preparation, and after frozen storage for 1, 3, 5 and 10 weaks.

\section{Results}

The results obtained are shown in Tables 4 (on BS suspension) and 5 (on fish meal). As can be seen in these Tables, the cell numbers of Salmonella in the two types of suspensions, in BS and fish meal, tended to decrease with the lapse of frozen storage time, and the decreasing rates in the BS suspension were more conspicuous than those in the fish meal, viz., the initial viable counts of Salmonella in the BS suspension ranged from $2.4 \times 10^{4} / \mathrm{m} l(0.1 \% \mathrm{PW})$ to $3.5 \times 10^{3} / \mathrm{m} l(\mathrm{BSM}$ and Sel), and after 10 weeks storage at $-20^{\circ} \mathrm{C}$ these decreased to $7.9 \times 10^{\circ} / \mathrm{m} l$ (BSM) to $8.0 \times 10^{-1} / \mathrm{m} l$ (Sel), while the initial counts in the fish meal samples ranged from $9.2 \times 10^{3} / \mathrm{g}(1 / 2 \mathrm{PBW}, \mathrm{EEM}$ and $0.1 \% \mathrm{PW}$ ) to $2.4 \times 10^{3} / \mathrm{g}$ (Sel), and these decreased to $2.4 \times 10^{2}(0.1 \% \mathrm{PW})$ to $3.3 \times 10 / \mathrm{g}$ (BPT and Sel).

It was found that $\mathrm{BSM}, 0.1 \% \mathrm{PW}$ and $\mathrm{NB}$ gave relatively high recoveries in Salmonella counts in the buffered saline solution and fish meal samples. In contrast, the direct selenite broth method that selenite broth and DHL plate are employed with- 
Table 5. Changes in the counts of S. typhimurium during frozen storage in fish meal sample, and comparison of pre-enrichment media for the recoveries of test organism

\begin{tabular}{|c|c|c|c|c|c|c|}
\hline & \multirow{2}{*}{ Medium } & \multicolumn{5}{|c|}{ Storage at $-20^{\circ} \mathrm{C}$} \\
\hline & & Initial*1 & 1 week & 3 & 5 & 10 \\
\hline 1. & $\mathrm{BSM}$ & $5.4 \times 10^{3 * 2}$ & $1.3 \times 10^{3}$ & $1.1 \times 10^{3}$ & $4.9 \times 10$ & $1.4 \times 10^{2}$ \\
\hline 2. & $1 / 2 \mathrm{BPW}$ & $9.2 \times 10^{3}$ & $7.0 \times 10^{2}$ & $4.9 \times 10^{2}$ & $1.7 \times 10^{2}$ & $7.9 \times 10$ \\
\hline 3. & $\mathrm{BPT}$ & $9.2 \times 10^{3}$ & $3.3 \times 10^{2}$ & $1.7 \times 10^{2}$ & $7.0 \times 10$ & $3.3 \times 10$ \\
\hline 4. & EEM & $9.2 \times 10^{3}$ & $1.7 \times 10^{2}$ & $2.3 \times 10^{2}$ & $7.0 \times 10$ & $4.9 \times 10$ \\
\hline \multirow[t]{2}{*}{5.} & Selenite & & & & & - \\
\hline & $\begin{array}{l}\text { Broth }\left(35^{\circ} \mathrm{C}\right. \\
\text { Direct })\end{array}$ & $2.4 \times 10^{3}$ & $1.7 \times 10^{2}$ & $5.0 \times 10$ & $1.4 \times 10$ & $3.3 \times 10$ \\
\hline 6. & NB & $5.4 \times 10^{3}$ & $7.9 \times 10^{2}$ & $1.1 \times 10^{3}$ & $3.3 \times 10^{2}$ & $1.1 \times 10^{2}$ \\
\hline 7. & $0.1 \% \mathrm{PW}$ & $9.2 \times 10^{3}$ & $3.3 \times 10^{2}$ & $4.6 \times 10^{2}$ & $2.4 \times 10^{2}$ & $2.4 \times 10^{2}$ \\
\hline
\end{tabular}

*1 A $0.2 \mathrm{ml}$ of $S$. typhimurium bacterial soultion containing $9.2 \times 10^{5}$ cells (MPN in Nutrient broth) was added to $20 \mathrm{~g}$ of fish meal. *2 Number of bacterial cells (MPN/g).

out pre-enrichment culturing, gave apparently poor recoveries for the Salmonella. It was also found that both BPT or EEM gave rather poor recoveries as compared with $\mathrm{BSM}, 0.1 \% \mathrm{PW}$ and NB.

\section{Discussion}

In Japan, both BPT and EEM are commonly employed as pre-enrichment media for Salmonella, particularly EEM has been adopted in the recommended procedure for the examination of Salmonella in food, and food poisoning specimens, ${ }^{8)}$ while BPT has been employed mainly for the tests of veterinary samples and animal feeds. ${ }^{8)}$ However, it was revealed in the present work (Experiment I) that BS gave the highest detection rates of salmonellae in fish meal samples among the three different media tested. The reason for the highest detection rates of BS as a pre-enrichment culture of salmonellae in fish meal samples would be as follows: Different from the BPT and EEM, BS does not contain any bacteriostatic agents which may be effective to eliminate other contaminants from salmonellae, but the presence of these agents or excessive nutrients may sometimes interfere with the growth of salmonellae, especially when the bacterial number in a test material is extremely low.

The same reason might be applicable to the results of the pre-enrichment media for quantitative test. Experiment II was done to compare effectiveness of pre-enrichment media for the isolation of Salmonella from fish meal samples under frozen conditions. In the ordinary commercial process, fish meal is never stored under frozen conditions mainly due to the economical reason, in addition, a product may rapidly absorb moisture immediately after taking off it from cold storage. In the present study, however, the fish meal samples were stored at $-20^{\circ} \mathrm{C}$ for $7-10$ days in order to prevent from the mold growth. Salmonella positive samples in the qualitative test, are generally subjected to the quantitative test. As shown in Tables 4 and 5, EEM, BPT and selenite broth gave apparently poor recoveries as compared with other media. The reason for the lower recoveries would be due to that these media contained some bacteriostatic agents such as cholic acid, selenite or some surfactants, in addition, these media are fairly rich in sugars and other nutrients. On the contrary, BSM, $0.1 \% \mathrm{PW}$, $1 / 2$ BPW and NB do not contain aforementioned agents, but are consisted of simple or rather poor nutrients. As a pre-enrichment medium for isolation of salmonellae from fish meals, it is preferable that the medium does not contain any bacteriostatic agents, in addition, the component should be simple with less nutrients. In addition to the aforementioned reasons, BS is employed as a common diluent in the field of food microbiology, and the author has adopted the BSM as the pre-enrichment medium in the quantitative test.

As shown in Table 2, Hajna's tetrathionate broth gave much higher detection rates than selenite broth, however, as for the pre-enrichment medium in the quantitative test, it requires more severe selectivity, and results of Experiment I indicated that the selenite broth would be the most appropriate enrichment medium for the aforementioned purpose. Based on the aforementioned findings, the author wishes to propose the following combined procedures for the examina- 
tion of salmonellae in fish meal samples; viz., as for the qualitative test, buffered saline solution (BS) should be used as a pre-enrichment medium, and the Hajna's tetrathionate broth as a selective enrichment medium, and as for the quantitative test, the buffered saline solution containing $0.1 \%$ meat extract (BSM) should be employed as a pre-enrichment medium.

\section{Acknowledgement}

The author wishes to express his deepest appreciation to Dr. T. Kawabata (National Institute of Health, Tokyo) for his kind advices and heartfelt encouragements. The author also grateful to Dr. T. Kawanishi (Japan Food Hygiene Association, Tokyo) for his collaboration in the serological analysis of the isolates. Thanks are also due to Dr. K. Hashimoto (National Institute of Animal Health, Tsukuba) for his useful advices. The author wishes to thank to Professors K. Hashimoto (University of Tokyo), S. Horie and M. Okuzumi (Tokyo University of Fisheries) for their encouragements.
This investigation was partly supported by the Grants from the Ministry of Education in Japan, and from the Towa Foundation for Food Research Promotion.

\section{References}

1) A. Granville: Technical Report Series (I.A.E.A.) No. 22, 9-33 (1963).

2) J. E. Williams: Wld. Poult. Sci. J., 37, 6-25 (1981).

3) Y. Asakawa, S. Akahane, and K. Shiozawa: Bull. Shizuoka Pref. Inst. Publ. Hlth., No. 23, 37-40 (1980).

4) Japanese Association of Food Hygiene: Shokuhin Eisei Kensa Shishin (Standard methods for the examination of foods) I. 93 (1973).

5) W. Edel, and E. H. Kampelmacher: Bull. Wld. Hlth. Org., 48, 167-174 (1973).

6) Ministry of Agriculture, Forestry and Fisheries, Japan: Atarashii Gijutsu (New technology), No. 18, 6-10 (1980).

7) Eiken Co.: Eiken Manual, 9th Ed., (1977).

8) A. Suzuki, T. Kawanishi, H. Konuma, S. Takayama, C. Imai, and J. Saitoh: J. Food Hygienic Soc. Jap., 22, 223-232 (1981). 